\title{
Financial Implications of Femtocell Deployment
}

\author{
Jana Zausinová1, Martin Zoričak,a, Gabriel Bugár² and Juraj Gazda² \\ ${ }^{1}$ Technical University of Košice, Faculty of Economics, 04001 Košice, Slovakia \\ ${ }^{2}$ Technical University of Košice, Faculty of Electrical Engineering and Informatics, 04001 Košice, Slovakia
}

\begin{abstract}
Increased usage in wireless communication has been observed in the last decades and it is expected to rise even more. Traditional spectrum allocation mechanism together with increasing demand for data transfers caused spectrum to become more congested. As a consequence, high pressure for its more effective usage emerges. Modern concept of heterogeneous networks with cognitive femtocells represents one of the promising solutions. Associated technical issues of heterogeneous networks have been discussed in many papers. However, economic aspects of femtocell deployment in network are still insufficiently analysed. This paper is devoted to economic aspects of operators' behaviour in the macro-femto network but we also focus on technical issue of overall spectrum usage. For this purpose, the agent-based model of two-tier network was proposed. Results of simulation confirm significant influence of number of deployed femtocells and their location in the network on operators' pricing strategies and their whole economic performance.
\end{abstract}

\section{Introduction}

With technological progress demand for frequency spectrum has been increasing for years. It creates pressure on spectrum usage efficiency, which is relatively low. Another issue is uneven spatial distribution of users. According to [1] around $60 \%$ of all users are located indoors, where macrocells coverage is considerably degraded. There have been many proposals for two-tier networks comprised of macrocells and femtocells. Such type of network offers benefits not only for users by increasing quality-of-service (QoS) but also for macrocell operators by offloading their traffic. However, proposed solution is accompanied by issues such as interference which was addressed by [2], [3] and [4] from technical point of view. Coexistence of macrocell and femtocells is considered in three basic modes: open, closed and hybrid [5]. Closed mode allows only registered users to connect, thus provides privacy and security for information transferred through network. Drawbacks of closed mode are potential cross-tier and co-tier interferences and lower overall throughput compared to open mode. Open mode is suitable for public areas to increase overall throughput. Negative aspects of open mode are potential degradation of QoS due to large number of users sharing limited sources, numerous handovers among femtocells or between femtocell and macrocell, and security issues. For private purposes optimal mode is hybrid, which allows access only limited number of unregistered users. Thus, hybrid mode provides relatively stable QoS for registered femtocell users.

Some of financial aspects of two-tier networks were analysed in several papers. Authors in [6] focused on

\footnotetext{
a Corresponding author: martin.zoricak@tuke.sk
}

pricing issues and introduced pricing models based on game theoretic framework. Proposed iterative gradient decent algorithm proved to be effective in quick convergence to Nash equilibria for both macrocell operator (MCO) and femtocell operators (FCOs). For solving pricing problem between $\mathrm{MCO}$ and FCOs authors in [7] employed auction mechanism, which is highly organized and efficient form of allocation of scarce resources. Knapsack algorithm was used in order to find optimal strategy leading to profit maximization for both MCO and FCOs. Another approach was used by authors in [8] to motivate FCOs to adopt hybrid mode. Opposed to previous studies, in this study authors consider MCO incentivizes FCOs to provide service to MCO users. Stackelberg game is used to maximize utilities of both tiers. Existing studies relay on assumption of perfect rationality and full information which is not realistic. Therefore, for our study we used Agent-based model, where rationality of agents is bounded.

\section{Model}

\subsection{Model preliminary}

The agent-based model was proposed to study pricing strategies of service providers, their interactions with endusers and other economic aspects of modelled system. The concept of two-tier macro-femto network considers three different types of agents: a) macrocell operator (MCO), b) femtocell operators (FCOs) and c) mobile end-users. MCO represents traditional wireless service provider that owns all of the available spectrum in the network and provides 
mobile services to end-users with deployed macrocell base station (MBS). On the other hand, FCOs have no own spectrum and have to lease it from MCO on the wholesale market when want to make profit and serve end-users. $\mathrm{MCO}$ has an incentive to share spectrum with FCOs when the reduction in its profit from providing services to endusers is fully compensated by profit from leasing spectrum. To accomplish that, the spectrum leasing price $w$ is set by $\mathrm{MCO}$ and this price influences behaviour of FCOs, especially their pricing strategies.

The proposed model of heterogeneous macro-femto network can be divided into two parts: the wholesale and the retail market. Available spectrum resources are dynamically leased by FCOs for fixed leasing price per resource block $(\mathrm{RB})$ on the wholesale market and both $\mathrm{MCO}$ and FCOs provide services to mobile end-users on the retail market. When femtocell and macrocell networks are not operated by the same operator, we can talk about competition on the retail market. In this case, network operators serve the same pool of end-users and try to maximize their own profits through dynamically adjusting own retail price. Choosing optimal pricing strategy that maximizes profit and responses to changing demand in the retail market is possible through implementation of learning algorithm. The approach of Linear rewardinaction was used for this purpose [9].

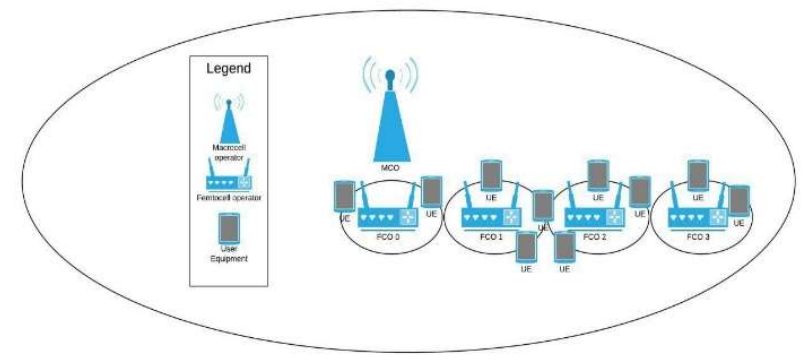

Figure 1. Schematic diagram of the network model

In our model, we consider one MBS located in the region operated on licensed spectrum and within the coverage area of MCO are located multiple FCOs in different distances from MBS. Different location of FCOs leads to different demand on the retail market. For eliminating inter-system interference, femtocell and macrocell base stations operate in separate spectrum [10]. In such spectrum access model, spectrum is split according to the number of participating femtocell providers and there is no correlation between channel quality under macrocell and femtocell. End-users are randomly distributed in the region. The end-users within coverage area of femtocell base station are able to access the femtocell but also macrocell network, following the "open access model". The end-users' decisions about accessing is affected by signal quality and also by price for access required by operators. The illustrative structure of the network model is depicted in Figure 1.

\subsection{Operators}

Our model contains two different types of operators. Via wholesale market MCO sell out a certain part of own licensed spectrum RBs and the rest part is kept for serving. For simplicity, we consider fixed number of total available RBs owned by MCO that has privilege to decide about part of RBs that will be leased to FCOs. The part of RBs allocated to FCOs is proportionally distributed among them. Each combination of RBs distribution between MCO and FCOs represents one of twelve analysed scenarios.

Let $B W$ be the number of available RBs owned by the MCO. At the beginning of the trading day, the MCO decides to allocate $s^{f}$ share of its $B W$ to FCOs, while $s^{f} \in$ $\{0,0.09,0.18, \ldots, 0.82,0.91,1\}$ including possibility not to allocate RBs to FCOs at all. On the other hand, the rest portion of $B W$, denotes as $s^{m}$, is kept for providing services to end-users, while $s^{f}+s^{m}=1$. There are $n$ FCOs operated in the network and each of them obtains $b_{i}$ RBs for leasing price $w$ per RB, while

$$
b_{i}=\left\lfloor\frac{s^{f} B W}{n}\right\rfloor .
$$

The spectrum leasing price $w$ is in our model fixed and is set by $\mathrm{MCO}$ that through this price can influence demand of FCOs for his licensed RBs and also control competition on the market. Leasing price $w$ represents lower limit for FCOs's retail prices $p_{i}^{f}$, that should not be less than $w$ in order to ensure the FCOs achieve a nonnegative profit. Primary goal of both operators is to maximize their profits. However, the market demand is uncertain as a result of cognitive capabilities of end-users' devices and network switching technology. As the consequences, operators have to dynamically plan a sufficient number of RBs in advance and adapt prices in real-time to accommodate demand in the retail market. To accomplish that, the pricing method called Linear rewardinaction was adopted. It is one of the most powerful tools from adaptive learning area related to the field of learning automata and belongs to probability-vector approaches. The length of this vector containing non-negative numbers that sum to 1 , corresponds to the number of possible pricing strategies of an agent. Agent chooses one of strategies according to a probability vector, which at every instant contains the probabilities of choosing each action. Crucial issue is the updating of probabilities. One of popular possibilities is of linear reward-inaction algorithm that has desired absorbing effect (wrong strategy is not executed with non-zero probability) because a probability is only increased by chosen strategy which succeeds. At the start of the simulation, the probability distribution of the strategies $\rho_{i}$ is uniform (i.e., $\rho_{i}(j)=0.1$ for all $j$ ). It is iteratively updated according to the Equation 2 until the increment in the probability vector is negligible and the learning process stops.

$$
\rho_{i}^{+1}(k)= \begin{cases}\rho_{i}(k)-\eta R_{i}(k) \rho_{i}(k) & \text { if } k \neq j \\ \rho_{i}(k)+\eta R_{i}(k) \sum_{k \neq j} \rho_{i}(k) & \text { if } j=k,\end{cases}
$$

where $\eta$ is learning parameter $(\eta \in\langle 0,1\rangle)$ and $R_{i}(k)$ represents the payoff of the $i$-th operator received by playing the $k$-th strategy. Payoffs represent the response (i.e., reward) to the corresponding action from the 
stochastic environment and help to choose the subsequent action. Through a series of interactions with environment, the provider finally approaches to optimal behaviour.

The payoff of MCO is calculated as follows

$$
R^{m}=\sum_{j \in C_{i}} p_{j}^{m}+\sum_{i=1}^{n} b_{i} w,
$$

where $C_{i}$ denotes number of end-users connected to the $\mathrm{MCO}, p^{m}$ represents retail price paid by $j$-th end-user to MCO for one-hour connection. On the other hand, the revenue of FCO is expressed as

$$
R_{i}^{f}=\sum_{j \in C_{i}} p_{i, j}^{f}-b_{i} w,
$$

where $C_{i}$ denotes number of end-users connected to the $i$ th $\mathrm{FCO}, p_{i, j}^{f}$ is the unit retail price paid by $j$-th end-user to $i$-th FCO for one-hour connection and $k_{i}$ represents number of leased RBs by $i$-th FCO.

\subsection{End-users}

The transactions between service providers and end-users are carried out on the retail market. The primary concept of this market is derived from [11]. Both operators offer spectrum access to end-users but for different prices. Endusers located within coverage area of one of femtocell operators is able to choose between different network operators. Accessing the femtocell network can provide higher data rate thanks to the shorter distance but required price per connection by FCO can be higher because it should compensate FCO's costs in form of spectrum leasing price. On the other side, network access price set by MCO can be lower but end-users can achieve lower data rate. Modelled end-users behave like rational entities. Through their ability to capture QoS and required price per connection, they want to achieve the highest possible utility. QoS achieved by end-users depends on the spectral efficiency of transmission that reflects influence of location and also signal power, and is expressed as

$$
r_{i, j}=\log _{2}\left[1+\frac{P_{s}}{N_{0}}\left(\frac{d_{i, j}}{\frac{L}{4}}\right)^{-2}\right],
$$

where $N_{0}$ is the additive white Gaussian noise (AWGN) variance, $P_{s}$ is the signal power, $d_{i, j}$ is the distance between the $i$-th end-user and base station of the $j$-th operator, and $L$ is the total length of the linear region ( $L=$ $1000 \mathrm{~m}$ in our simulation setup). The base station of FCO has lower transmission power but it benefits from shorter distance from end-users. Used utility function of end-users depends on spectral efficiency, the utility is increasing with increasing spectral efficiency, see Equation 6.

$$
U_{i, j}=e^{-\alpha\left(\frac{1}{r_{i, j}}\right)^{\beta}},
$$

where $\alpha, \beta$ are utility function parameters that set the shape of the utility function. Also the sensitivity to a price for one-hour connection $p^{m}, p_{i}^{f}$ has to be implemented. To accomplish that, each end-user has assigned acceptance probability $A_{i, j}$ that expresses the probability to accept an offer of the $j$-th operator given by following formula

$$
A_{i, j}=1-e^{-c U_{i, j}^{\delta}\left(1-p_{i, j}\right)^{\gamma}},
$$

where $c$ is an appropriate constant and $\delta, \gamma>0$ are the parameters describing the sensitivity of the end-user to both utility and price, respectively.

\section{Simulation results}

Simulations of our model were conducted in software designed for agent-based modelling - NetLogo. Each of them consists of 100000 simulation rounds (so called ticks) while first 40000 rounds were disregarded to ignore nonstationary development. One tick represents one day, while minimum connection length is one hour and transaction on the retail market run once an hour. For getting robust results, each simulation was run ten times with the same parameters settings and the results were averaged. The settings of used parameters are shown in Table 1. Twelve different combinations of RBs distribution among operators and scenarios with 2, 3 or 4 FCOs located in various distance from MBS were analyzed.

Table 1. Parameters settings

\begin{tabular}{|l|c|c|}
\hline \multicolumn{1}{|c|}{ Parameter } & Symbol & Value \\
\hline Number of FCOs & $\mathrm{n}$ & $\{2,3,4\}$ \\
\hline Number of end-users & - & 100 \\
\hline Leasing spectrum price & $\mathrm{w}$ & 0.2 \\
\hline Starting retail price & $\mathrm{p}$ & 0.02 \\
\hline Utility function parameter & $\alpha$ & 0.01 \\
\hline Utility function parameter & $\beta$ & 0.8 \\
\hline $\begin{array}{l}\text { Acceptance probability } \\
\text { function parameter }\end{array}$ & $\gamma$ & 2.4 \\
\hline $\begin{array}{l}\text { Acceptance probability } \\
\text { function parameter }\end{array}$ & $\delta$ & 0.55 \\
\hline $\begin{array}{l}\text { Acceptance probability } \\
\text { function parameter }\end{array}$ & $\mathrm{c}$ & 8 \\
\hline $\begin{array}{l}\text { Linear reward-inaction } \\
\text { learning parameter }\end{array}$ & $\eta$ & 0.004 \\
\hline Number of pricing strategies & - & 10 \\
\hline
\end{tabular}

Spectrum usage is one of the most important indicators because of the frequency spectrum scarcity. The main benefit from introduction of heterogeneous macro-femto network is an increase in spectrum usage. According to the results of our simulations depicted in Figure 2, spectrum usage is significantly influenced by chosen distribution of RBs among operators. Overall spectrum usage is maximized when $36 \%$ - $55 \%$ of total available RBs owned by MCO are allocated to FCOs when we consider constant number of end-users distributed in the region.

Higher number of femtocell operators enables to achieve higher spectrum usage, but this increment in spectrum usage disappears when number of FCOs operating in the network is more than three, under 
unchanged demand. Benefits from increment in spectrum usage have to be evaluated in terms of additional costs that are incurred by deployment of new FCO in the network. The same or even higher usage than in a case when are deployed all four FCOs, can be achieved when only three FCOs (i.e., $\mathrm{FCO}_{0}, \mathrm{FCO}_{1}, \mathrm{FCO}_{2}$ ) are deployed in the region.

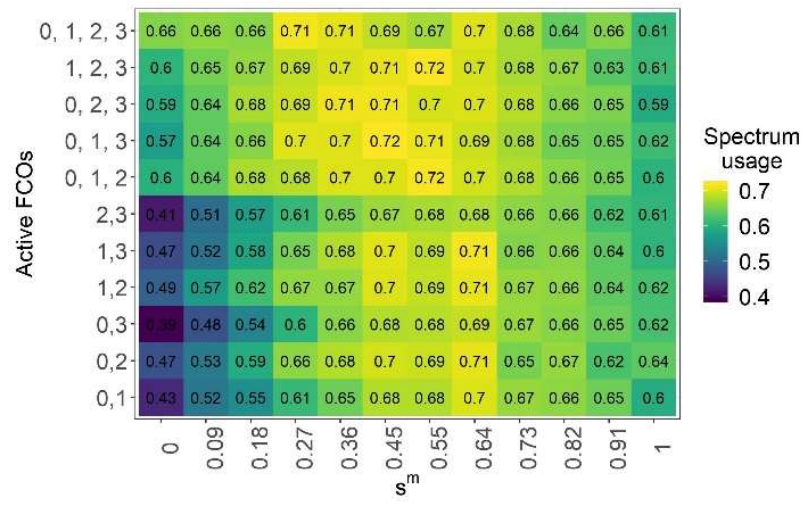

Figure 2. Heat map representing spectrum usage related to $\mathrm{s}^{\mathrm{m}}$ and deployment of FCOs

Overall spectrum usage is also influenced by location of operating FCOs. The situation when there are only two active FCOs and they are located too close to MBS or, vice versa too far at the edge of region, the spectrum is not used very efficiently. Better solution represents scenario with one FCO at the edge and another located closer to MCO. Such deployment of FCOs can increase service quality by offloading from MCO and increase capacity at cell edges where performance can significantly degrade. When FCOs are located too close to each other and large area of region remains in the competence of $\mathrm{MCO}$, benefits from FCOs' deployment are not very significant.

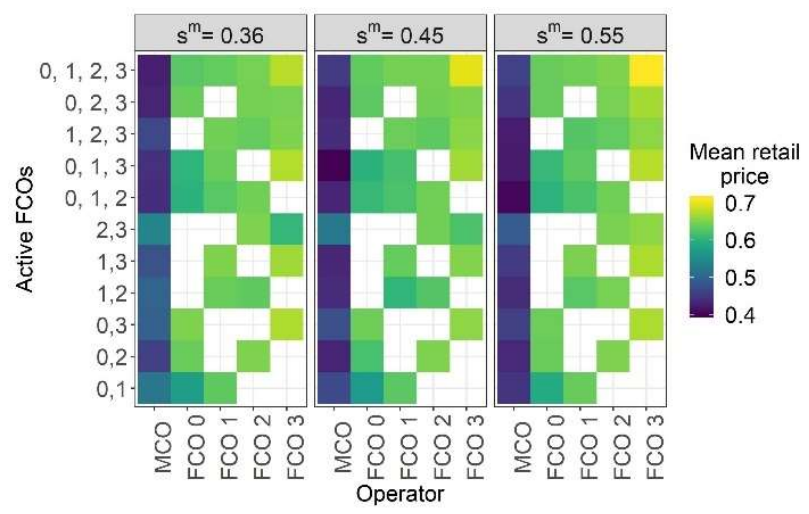

Figure 3. Heat map representing retail prices related to $\mathrm{s}^{\mathrm{m}}$ and deployment of FCOs

Crucial for both operators is choosing optimal pricing strategy that maximizes their profit. Depicted distributions of available RBs between MCO a FCOs in Figure 3, represent the most efficient ones in term of overall spectrum usage. The impact of different levels of supply on pricing strategies under unchanged demand can be observed by comparing these individual divisions. Increase in portion of RBs retained by causes that its supply exceeds demand even more and it leads, by basic economic rules, to reduction of MCO retail price. This fact is not so significant in case of FCOs, whose prices are also affected by location of other FCOs and their own distance from MBS. More FCOs in the region creates higher competition for MCO and this fact leads to lower MCO's retail price in scenarios with three or four FCOs. Competition among FCOs appears more obviously in case when they are located closer to each other and compete for the same pool of end-users. Retail price of FCO located farthest form the MBS is highest and is negatively correlated by distance to nearest FCO.

Chosen pricing strategy is reflected in mean daily profits of FCOs (see Figure 4). With decreasing number of leased RBs, the mean daily profit per FCO also decreases. Also higher number of existing operators in the market reduces the possibility of achieving high profits. FCOs located farther from the MBS and those without neighboring FCOs achieve higher profit as a consequence of reduced competition. They are able to meet larger part of demand in the market and set higher retail price without decreasing their attractiveness to end-users.

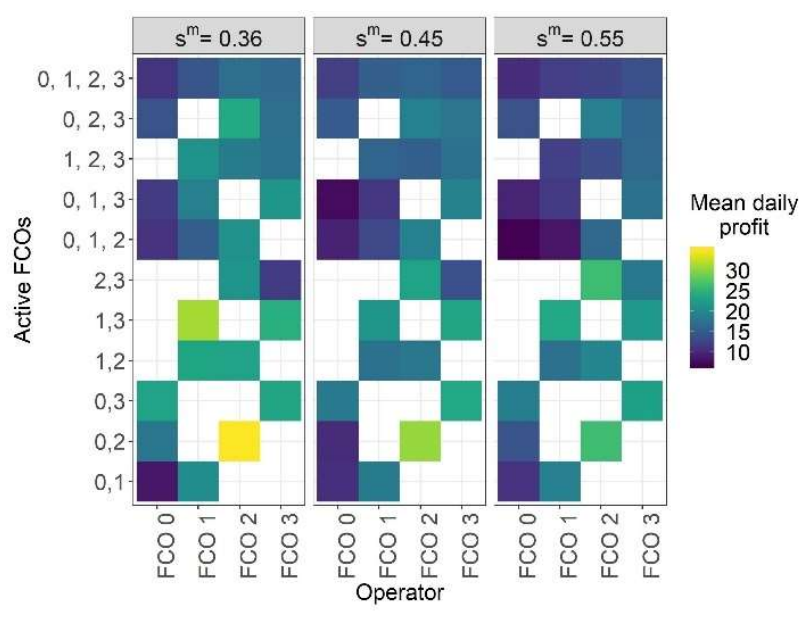

Figure 4. Heat map representing daily profit related to $\mathrm{s}^{\mathrm{m}}$ and deployment of FCOs

Importance of femtocell operators is mainly visible in the most crowded areas and also at cell edges. Adding femtocells can improve network performance and service quality by offloading of traffic. To analyse this potential of heterogeneous network we created modified model containing areas with lower demand density near MCO and at the edge of cell and higher demand density area in the between. Various combinations of locations of two FCOs was studied and the results are depicted in Figure 5.

Overall spectrum usage is significantly influenced by location of FCOs. According to the results of our simulations, the highest spectrum usage can be reached by locating FCOs in areas with high demand density. This spectrum usage is even higher than in situation with deployment of four FCOs. On the other hand, in a case when FCOs are located only in regions where the endusers are sparsely concentrated, they are not able to improve utility for sufficient number of end-users and can not contribute to better spectrum usage. 


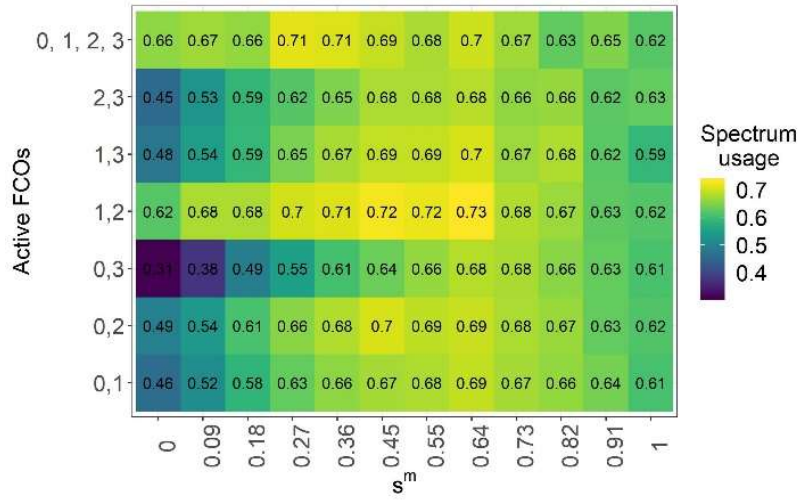

Figure 5. Heat map representing spectrum usage related to $\mathrm{s}^{\mathrm{m}}$ and deployment of FCOs in areas with different demand density

\section{Conclusions}

In this paper was analyzed impact of number of FCOs and their location in network on overall spectrum usage and various economic aspects of heterogeneous networks such as pricing strategies and corresponding profits of individual operators. For this purpose, the agent-based model of heterogeneous macro-femtocell operators was proposed. Results of our simulations confirm that number of deployed FCOs significantly influences overall spectrum usage. The location of FCOs seems to have even greater impact on usage and also the importance of FCOs in the most crowded areas and at edges of the cells has been confirmed. We have shown effect of analyzed factors on choosing of optimal pricing strategies of operators and influence of competition among them. Simulations were based on two assumtions: the femtocell and macrocell are individual operators and fixed leasing spectrum price was set. In the future, we will study the hybrid model with femtocell and macrocell operated by same service provider and dynamic allocation of RBs among operators through auction market mechanism.

\section{Acknowledgements}

This work was supported by the Slovak Research and Development Agency, project number APVV-15-0358.

\section{References}

1. S. Al-Rubaye, A. Al-Dulaimi and J. Cosmas, "Cognitive femtocell," IEEE Vehicular Technology Magazine, vol. 6, pp. 44-51, 2011.

2. J. Espino and J. Markendahl, "Analysis of macrofemtocell interference and implications for spectrum allocation," in 2009 IEEE 20th International Symposium on Personal, Indoor and Mobile Radio Communications, 2009.

3. S. Jafarpour-Alamdari, V. Solouk, H. Kalbkhani and M. G. Shayesteh, "Interference analysis of femto-macrocell networks in 6-sector macrocell layout," in 6th International Symposium on Telecommunications (IST), 2012.

4. S. Jain and M. Hussain, "Improving Capacity in Wireless Heterogeneous Networks by Mitigation of Interference," Procedia Computer Science, vol. 115, pp. 596-606, 2017.

5. S. Padmapriya and M. Tamilarasi, "A case study on femtocell access modes," Engineering Science and Technology, an International Journal, vol. 19, pp. 1534-1542, 2016.

6. C. Jiang, Y. Chen, K. J. R. Liu and Y. Ren, "Optimal Pricing Strategy for Operators in Cognitive Femtocell Networks," IEEE Transactions on Wireless Communications, vol. 13, pp. 5288-5301, 92014.

7. X. Chen, L. Xing, T. Qiu and Z. Li, "An AuctionBased Spectrum Leasing Mechanism for Mobile Macro-Femtocell Networks of IoT," Sensors, vol. 17, p. 380, 22017.

8. L. Li, M. Wei, C. Xu and Z. Zhou, "Rate-Based Pricing Framework in Hybrid Access Femtocell Networks," IEEE Communications Letters, vol. 19, pp. 1560-1563, 92015.

9. Y. Xing, R. Chandramouli and C. Cordeiro, "Price dynamics in competitive agile spectrum access markets," IEEE Journal on Selected Areas in Communications, vol. 25, pp. 613-621, 42007.

10. C. Y. Wang and H. Y. Wei, "Profit Maximization in Femtocell Service with Contract Design," IEEE Transactions on Wireless Communications, vol. 12, pp. 1978-1988, 52013.

11. J. Gazda, V. Kováč, P. Tóth, P. Drotár and V. Gazda, "Tax optimization in an agent-based model of real-time spectrum secondary market," Telecommunication Systems, vol. 64, pp. 543558, 2017. 